\title{
AVALIAÇÃO PELO P300 DE CRIANÇAS COM E SEM EPILEPSIA E RENDIMENTO ESCOLAR
}

\author{
JUCELEI F. VISIOLI-MELO*; NEWRA TELLECHEA ROTTA**
}

\begin{abstract}
RESUMO - Dificuldade de aprendizagem é situação comum em crianças com epilepsia. Distúrbios da inteligência têm sido associados com epilepsia. O potencial cognitivo (P300) é um adjunto clínico para mensurar neurofisiologicamente o processo cognitivo. Foram estudadas 99 crianças com 10 anos a 11 anos e 11 meses. Do Grupo I, sem epilepsia, faziam parte 64 crianças, das quais 32 com bom rendimento e outras 32 com mau rendimento escolar. Do Grupo II, com epilepsia, faziam parte 35 crianças, sendo 21 com bom rendimento escolar e 15 com mau rendimento escolar. Não foi encontrada diferença significativa na latência do P300 entre os dois grupos. Quando foram estratificados segundo o desempenho escolar, as crianças do Grupo I, com bom rendimento escolar, apresentaram latência do P300 de 336 ms e as com mau rendimento escolar, latência de 382 ms; as crianças do Grupo II, com bom rendimento escolar, apresentaram latência do P300 de $363 \mathrm{~ms}$ e as com mau rendimento escolar, latência de $400 \mathrm{~ms}$, com diferença significativa. Essa diferença estava localizada entre as crianças não epilépticas com bom desempenho escolar e as com mau desempenho escolar, epilépticas ou não.
\end{abstract}

PALAVRAS-CHAVE: epilepsia, potencial evocado, P300, cognição, rendimento escolar.

\section{Assessment through P300 of epileptic and non-epileptic children and school performance}

ABSTRACT - Learning disability is common in epileptic children. Epilepsy has been associated with disorders of intelligence. Cognitive potential (P300) is considered to be a clinical aid in the neurophysiological measurement of the cognitive process. Ninety-nine children between the ages of 10 years and 11 years and 11 months formed our sample, with good and poor school performance. Group I, non-epileptic, had 64 children of whom 32 had good and 32 poor school performance. Group II, epileptic, had 35 children, of whom 21 had good and 15 poor school performance. No significant difference in P300 latency was found between Groups I and II. When groups were stratified based on school performance, Group I children with good school performance had P300 latency of $336 \mathrm{~ms}$, while the ones with poor school performance had latency of $382 \mathrm{~ms}$. Group II children with good school performance had P300 latency of 363, while the ones with poor school performance had latency of 400 $\mathrm{ms}$. There was a statistically significant difference between the non-epileptic children with good school performance and the children with poor school performance, epileptic or not.

KEY WORDS: epilepsy, evoked potentials, P300, cognition, school performance.

As primeiras observações sobre a atração de corpos eletrizados remontam à Grécia antiga com o filósofo Tales de Mileto. A partir do século XVIII foram sendo estudados os fenômenos elétricos vinculados ao sistema nervoso $(\mathrm{SN})$, inicialmente através dos efeitos da aplicação da eletricidade ao corpo e, após, através do próprio corpo como fonte de eletricidade ${ }^{1,2}$. Com o advento da computação, em 1951, Dawson pôde introduzir as bases para a obtenção dos potenciais evocados sensoriais (PES) que, na década de 70, foram reconhecidos como de utilidade clínica, após o desenvolvimento de amplificadores fisiológicos para os sinais elétricos captados ${ }^{3}$. A expressão

Curso de Pós-graduação em Pediatria da Universidade Federal do Rio Grande do Sul (UFRGS), Unidade de Neurologia Pediátrica do Hospital de Clínicas de Porto Alegre (HCPA): *Mestre em Pediatria, com concentração em Neurologia Pediátrica, UFGRS; **Professora Adjunta de Neurologia, UFGRS. Aceite: 24-março-2000.

Dra Jucelei Visioli Melo - Praça Júlio de Castilhos, 20/301 - 90430-020 Porto Alegre RS - Brasil. 
potencial evocado (PE) é usada especificamente para designar as respostas do sistema nervoso central (SNC) ao estímulo sensorial ou elétrico. O potencial de longa latência é uma série de ondas positivas e negativas que são geradas acima do tronco cerebral e com extensa distribuição no escalpo e com latência que varia de $250-600 \mathrm{~ms}^{4}$. O P300 deve ser obtido quando o paciente está acordado, alerta e distingue um estímulo combinado entre um grupo de estímulos, representando o período de resposta do córtex de associação ao estímulo do córtex primário ${ }^{5,6}$. O gerador ou os geradores neuronais específicos permanecem incertos ${ }^{7}$. Sua origem não é exclusivamente cortical, sendo também subcortical. Considera-se que o P300 tem correlação com o hipocampo, tálamo e lobo frontal ${ }^{8}$. Halgren et al. ${ }^{9}$, em estudos com humanos, sugeriram que atividade neural límbica estaria envolvida na geração do P300. A memória é um processo complexo, envolvendo subsistemas, e o P300 pode refletir um subcomponente do sistema. O P300 se constitui em uma oportunidade para investigar o mecanismo eletroquímico e o aspecto temporal da cognição, é referido como sendo uma função cognitiva, particularmente nas áreas relacionadas à atenção e à memória recente ${ }^{7}$. O P300 pode ser alterado por algumas características do sujeito entre as quais se incluem o nível de atenção, a dificuldade da tarefa proposta e a idade ${ }^{4,5,10-12}$. Foi encontrada significativa correlação do P300 com QI pelo teste de Wechsler Intelligence Scale for Children Revised (WISC-R) e pelo escore total da escala Wechsler, especialmente para os itens de atenção e memória recente ${ }^{12-14}$. Martin et al. ${ }^{14}$ também encontraram relação entre o componente P300 e habilidade cognitiva em crianças, usando a Terman Merril Scale para as crianças com menos de 4 anos e o WISC para crianças acima dessa idade.

O SNC desenvolve-se rapidamente na infância, época em que muitas epilepsias de início idade-dependente e com curso clínico durante a maturação, podem ser consideradas desordens do desenvolvimento ${ }^{13}$. A idade precoce do início das crises pode interferir no desenvolvimento cerebral e, por consequiência, provocar, a longo prazo, impacto na cognição por inibição na atividade mitótica, afetando a mielinização e reduzindo o número de células. Por isso é pior o prognóstico para o desenvolvimento cognitivo nas encefalopatias idade-dependente ${ }^{15}$. Seidenberg et al. ${ }^{16}$ correlacionaram a alta incidência de problemas escolares com epilepsia, sendo que crianças epilépticas apresentaram menor desempenho escolar. Drake et al. ${ }^{17}$ descreveram aumento da latência dos componentes do potencial cognitivo nos casos com epilepsia em relação aos não epilépticos. As mudanças de latência do $\mathrm{P} 300$ podem diferir conforme a síndrome epiléptica ${ }^{12,13}$.

O objetivo geral deste estudo foi verificar a repercussão da epilepsia em relação ao potencial cognitivo, P300. Os objetivos específicos foram definir a latência do P300 em crianças com idade entre 10 anos e 11 anos e 11 meses, estudar a relação aprendizagem escolar e P300, observar se a presença de alterações eletrencefalográficas interferem no resultado do P300 e determinar, com o estudo do P300, a repercussão da terapia anticonvulsivante.

\section{MÉTODO}

Foi realizado um estudo transversal, contemporâneo, por meio de uma amostragem de conveniência, selecionando crianças com 10 anos a 11 anos e 11 meses (de janeiro de 1996 a janeiro de 1998) com epilepsia entre aquelas que procuraram o Ambulatório de Neurologia Infantil do HCPA e crianças sem epilepsia encaminhadas de duas escolas estaduais. O desfecho do estudo foi o P300. Participaram do estudo 99 crianças. O tamanho da amostra calculado para testar uma diferença de $50 \mathrm{~ms}$ ou mais na latência do P300, maior que o desvio padrão, considerando a variável rendimento escolar, com alfa de $5 \%$ e poder estatístico de $90 \%$, foi 24 crianças epilépticas e 48 não epilépticas num total de 72 crianças.

Foram selecionadas por sorteio 64 crianças não epilépticas, (Grupo I), sendo 32 com bom rendimento escolar e outras 32 com mau rendimento escolar, tendo duas ou mais reprovações; e 35 crianças epilépticas (Grupo II), também estratificas quanto ao desempenho escolar. Todas as crianças foram submetidas a exame clínico-neurológico, a estudo do P300 e a exame eletrencefalográfico .

As variáveis quantitativas foram descritas com medidas de tendência central (média e mediana) e medidas de dispersão (DP). Para as variáveis categóricas foram usadas porcentagens. Para comparar os grupos, separados segundo os fatores epilepsia e rendimento escolar, utilizou-se o teste da ANOVA com localização das diferenças pelo teste de Tukey. Na comparação de dois grupos independentes utilizou-se o teste t de Student. As variáveis 
categóricas foram comparadas pelo teste qui-quadrado com correção de Yates ou pelo teste exato de Fisher, quando necessário. Nas situações de desvio da distribuição gaussiana, foram utilizados os testes não-paramétricos de Kruskall-Wallis e Mann-Whitney. O nível de significância utilizado foi $5 \%(\mathrm{p} \leq 0,05)$. Os dados foram processados e analisados com o auxílio dos programas Epi Info v6.0. e Statistical Package for Social Sciences (SPSS) v6.0.

\section{RESULTADOS}

O estudo baseou-se na avaliação de 99 crianças não havendo diferença significativa quanto ao rendimento escolar por apresentar ou não epilepsia.

A amostra constituiu-se de 37 (37\%) crianças do gênero feminino, das quais 24 pertenciam ao Grupo I e 13 ao Grupo II, e de 62 (63\%) crianças do sexo masculino, sendo que 40 pertenciam ao Grupo I e 22 ao Grupo II. Quanto ao desempenho escolar, 22 meninas apresentavam bom rendimento e 15 tinham mau rendimento; quanto aos meninos, 31 apresentavam bom rendimento e 31 tinham mau rendimento escolar.

No Grupo I, 11 mães eram analfabetas, 21 tinham o primeiro grau incompleto, 18 concluíram o primeiro grau, 9 haviam feito o segundo grau e 5 o terceiro grau. No Grupo II, 8 mães eram analfabetas, 16 tinham o primeiro grau incompleto, 3 haviam concluído o primeiro grau, 3 o segundo grau, 4 ingressaram no terceiro grau e em 1 caso a informação não foi obtida. Não ocorreu diferença significativa quanto a esse aspecto. No grupo das crianças com bom desempenho escolar, não foi obtida a informação sobre a escolaridade da mãe em 1 caso, 8 mães eram analfabetas, 16 tinham o primeiro grau incompleto, 10 completaram o primeiro grau, 11 haviam cursado o segundo grau e 7 ingressaram no terceiro grau. No grupo das crianças com baixo desempenho escolar, 11 mães eram analfabetas, 21 tinham o primeiro grau incompleto, 11 concluíram o primeiro grau, 1 o segundo grau e 2 o terceiro grau. $\mathrm{O}$ grau de escolaridade materna apresentou diferença significativa quanto ao rendimento escolar da criança $(\mathrm{p}=0,002)$. Não ocorreu diferença significativa quanto à escolaridade do pai. Foi analisada a renda per capita nos 2 grupos, não havendo diferença significativa. Quando foi considerada a renda familiar em relação ao rendimento escolar das crianças, houve diferença significativa quanto ao desempenho escolar $(\mathrm{p}<0,001)$.

Foram realizados eletrencefalogramas (EEG) em todas as crianças que participaram do estudo. Do Grupo I faziam parte 32 crianças com bom rendimento escolar, sendo que, 17 delas apresentavam EEG normal e 15, EEG alterado. As alterações eletrencefalográficas encontradas foram: paroxismos na região temporal em 2 exames, paroxismos centro-temporais em 11, paroxismos occipitais em $1 \mathrm{e}$ paroxismos multifocais em 1. Das 32 crianças que apresentavam baixo rendimento escolar, em $11 \mathrm{o}$ EEG foi normal e em 21 o exame estava alterado. Alterações generalizadas foram encontradas em 2 exames e focais em 19, sendo que paroxismos frontais foram vistos em 1, paroxismos temporais em 3 , paroxismos centro-temporais em 8 , paroxismos parietais em 1, paroxismos occipitais em 2 e paroxismos multifocais em 4. Não ocorreu associação entre o desempenho escolar e o EEG. Do Grupo II faziam parte crianças que tiveram suas crises com início após os 4 anos de idade. Nas 21 crianças com bom rendimento escolar, as alterações eletrencefalográficas identificadas foram: alterações generalizadas em 1 exame, focais em 16 e em 4 não foram identificadas alterações. As alterações focais observadas foram paroxismos frontais em 1, paroxismos temporais em 4, paroxismos centro-temporais em 7, paroxismos parietais em 1, paroxismos occipitais em 1 e paroxismos multifocais em 2. As alterações encontradas nas crianças com baixo rendimento escolar foram: 1 com paroxismos frontais, 2 com paroxismos temporais, $8 \mathrm{com}$ paroxismos centro-temporais e 3 com paroxismos multifocais. Não ocorreu diferença significativa quanto ao rendimento escolar da criança em relação ao tipo de EEG. A distribuição das crianças, segundo a classificação da epilepsia, para o grupo com bom rendimento escolar foi: 15 casos de epilepsia idiopática localizada e 6 casos de epilepsia idiopática generalizada. No grupo das crianças com baixo rendimento escolar, 9 apresentavam epilepsia idiopática localizada, 3 epilepsia idiopática generalizada e 2 epilepsia sintomática generalizada. O fator epilepsia não apresentou diferença significativa na variável rendimento escolar $(\mathrm{p}=0,35)$. Quanto ao uso de medicação, as crianças do Grupo II com bom 
rendimento escolar recebiam as seguintes DAE: 11 crianças CBZ, 3 VPA, 1 PB, 1 PHT, 4 OCBZ e 1 politerapia. As crianças com mau rendimento escolar recebiam: 7 crianças CBZ, 1 VPA, 3 PB e 3 politerapia .

A escolaridade da mãe não estava associada com a latência do P300. A renda per capita familiar também não provocou diferença significativa nas latências do P300.

Foi realizada a comparação entre as crianças do Grupo I quanto ao rendimento escolar. A média encontrada do P300 para as 32 crianças com bom rendimento escolar foi de $336 \mathrm{~ms}$, com desvio padrão de $48 \mathrm{~ms}$, para um intervalo de confiança de $95 \%$, sendo que variou de 318 a $353 \mathrm{~ms}$. Nas 32 crianças com baixo rendimento, a média do P300 foi de $382 \mathrm{~ms}$, com desvio padrão de 57 ms, para um intervalo de confiança de $95 \%$, sendo que variou de 361 a 403 ms. Não foi encontrada diferença significativa. A comparação entre as crianças do Grupo II, quanto ao desempenho escolar, mostrou que nas $21 \mathrm{com}$ bom desempenho a média da latência do P300 foi de $363 \mathrm{~ms}$, com desvio padrão de $52 \mathrm{~ms}$, para um intervalo de confiança de $95 \%$, tendo variado de 339 a $387 \mathrm{~ms}$. Nas 14 crianças com mau rendimento escolar, a média do P300 foi $400 \mathrm{~ms}$, com desvio padrão de $68 \mathrm{~ms}$, para um intervalo de confiança de $95 \%$, tendo variado de 360 a 439 ms. Não houve diferença significativa. Na comparação entre as médias do P300 das 32 crianças com bom rendimento escolar do Grupo I com latência de $336 \mathrm{~ms}$ com as das 21 crianças com bom rendimento escolar do Grupo II com latência de $363 \mathrm{~ms}$, não foi encontrada diferença significativa. Na comparação entre as médias do P300 das 32 crianças com baixo desempenho escolar do Grupo I com latência de $382 \mathrm{~ms}$ com as das 14 crianças do Grupo II com latência de 400 ms, não houve diferença significativa. Dividindo a amostra em crianças não epilépticas com bom e com mau desempenho escolar e crianças epilépticas com bom e mau desempenho escolar, foi observada diferença significativa $(\mathrm{p}<0,001)$ (Tabela 1$)$. Quando aplicados os testes estatísticos para localizar onde estaria essa diferença, foi observado que havia diferença significativa entre as crianças não epilépticas com bom desempenho escolar e as com mau rendimento, epilépticas ou não.

Foi considerada a latência do P300 como normal até 336 ms, pelo resultado obtido nas crianças não epilépticas com bom desempenho escolar. Na comparação entre não afetados, com resultado até este valor, e os afetados, com resultado acima deste valor, ocorreu diferença significativa $(\mathrm{p}=0,002)$ (Figura 1).

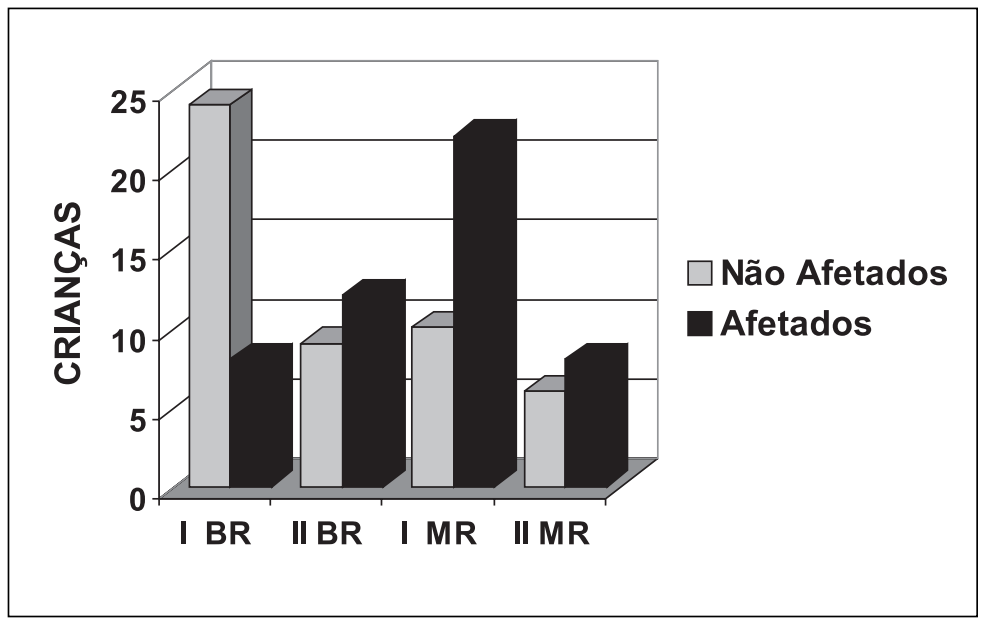

Figura 1. Latência do P300 segundo o desempenho escolar (n=99); $p=0,002$

$B R$, bom rendimento escolar; $M R$, mau rendimento escolar; Não afetados, até $336 \mathrm{~ms}$; Afetados, acima de $336 \mathrm{~ms}$ 
Tabela 1. Médias da latência do P300 nos dois grupos segundo o desempenho escolar

\begin{tabular}{lcc}
\hline Grupo & Bom Rendimento & Mau Rendimento \\
\hline I $(\mathrm{n}=64)$ & 336 & 382 \\
II $(\mathrm{n}=35)$ & 363 & 400 \\
\hline
\end{tabular}

$\mathrm{p}<0,001$

Tabela 2. Médias da latência do P300 no GRUPO I segundo desempenho escolar e EEG

\begin{tabular}{lcc}
\hline EEG & Bom Rendimento & Mau Rendimento \\
\hline Normal $(\mathrm{n}=28)$ & $336 \mathrm{~ms}$ (DP 53) & $399 \mathrm{~ms}$ (DP 49) \\
Com Paroxismos $(\mathrm{n}=36)$ & $335 \mathrm{~ms}$ (DP 44) & 373 ms (DP 59) \\
\hline
\end{tabular}

$\mathrm{p}=0,75$

DP, Desvio Padrão.

Considerando as variáveis EEG e P300 das crianças que não eram epilépticas, com bom e mau desempenho escolar, não foi encontrada diferença significativa quanto as latências do P300 entre as crianças que apresentavam EEG normal e EEG alterado. (Tabela 2).

As crianças que usaram medicação foram discriminadas pelas DAE, não ocorrendo diferença significativa. Tabela 3.

\section{DISCUSSÃO}

A escolaridade valoriza os aspectos intelectuais, determinando uma importância crescente para as ciências que avaliam a integridade do SN. Sinais cada vez mais sutis do comprometimento das funções cerebrais são diagnosticados ${ }^{18}$. Os distúrbios da inteligência têm sido associados com epilepsia a partir de uma ruptura na função cerebral, mas as inter-relações entre função cognitiva e condição epiléptica são extremamente complexas ${ }^{15}$.

Nos estudos da função cognitiva a falta de testes específicos, sensíveis e reproduzíveis pode propiciar variações na interpretação. Os testes neuropsicológicos traduzidos e publicados no Brasil são poucos e, na realidade, são testes de nível intelectual que fazem parte de baterias neuropsicológicas. O WISC é um teste de inteligência infantil editado em 1949 nos Estados Unidos, sendo o único das

Tabela 3. Médias da Latência do P300 segundo a DAE, Grupo II.

\begin{tabular}{lcc}
\hline DAE & $\mathrm{n}$ & Latência (ms) \\
\hline CBZ & 18 & 375 \\
VPA & 4 & 381 \\
PB & 4 & 383 \\
PHT & 1 & 396 \\
OCBZ & 4 & 356 \\
POLITERAPIA & 4 & 390 \\
\hline
\end{tabular}


escalas Wechsler publicado em português ${ }^{19}$. Contudo os testes de inteligência são importantes instrumentos de trabalho que, quando bem aplicados e, sobretudo, bem interpretados, permitem a avaliação das possibilidades, dos potenciais de inserção social da criança em determinados meios e orientam a abordagem terapêutica em função de suas dificuldades ${ }^{20}$. Estudos prévios descrevem correlação do P300 com testes psicológicos. Alguns autores se referem ao P300 como sendo mais sensível do que testes tipo WISC-R ou BENDER ${ }^{11-13,17}$.

Convulsões estão usualmente associadas com comprometimento transitório da função cognitiva e, portanto, do aprendizado; como ocorrem mais freqüentemente no período de desenvolvimento, é possível que estejam implicadas na etiologia do retardo mental. Também se faz necessário considerar as consequiências a longo prazo das DAE no desenvolvimento do cérebro ${ }^{21}$.

A idade tem efeito significativo em relação à memória e ao $\mathrm{P} 300^{7}$. Durante a infância, a latência do $\mathrm{P} 300$ decresce progressivamente no decorrer dos anos ${ }^{10}$. Esse decréscimo equipara-se à duração do desenvolvimento da memória, podendo estar relacionado com o fenômeno maturativo do processo cognitivo ${ }^{5,6,10-12}$. São poucos os trabalhos em que crianças na mesma faixa etária, em número expressivo, foram avaliadas com o P300, o que contribui para aumentar as dúvidas e controvérsias, até mesmo no que se refere a padrões de normalidade. Este é o primeiro estudo que avalia crianças epilépticas e não epilépticas com o P300 em nosso meio. O fato de que ocorre modificação da latência com a maturação cerebral fez com que se estudasse uma faixa etária definida. A escolha para esta pesquisa de crianças com idade de 10 a 11 anos e 11 meses corresponde à necessidade de relacionar o desempenho escolar quanto a ter duas ou mais repetências e também devido a serem 11 anos e 11 meses o limite da faixa etária atendida no Ambulatório de Neurologia Infantil do HCPA. O valor da latência do P300 obtido nesta pesquisa para as crianças não epilépticas com bom rendimento escolar apresentou valor próximo ao de Courchesne ${ }^{10}$, único trabalho encontrado que estudou a faixa etária semelhante à do presente estudo. Nesta pesquisa não ocorreu diferença significativa na latência do P300 entre meninos e meninas. Tais resultados concordam com relatos de Goodin ${ }^{6}$.

Os fatores sócio-culturais podem influenciar nos distúrbios cognitivos e do aprendizado ${ }^{16}$. Os fatores sócio-econômicos são os maiores preditores nos resultados dos testes de inteligência ${ }^{22}$. $\mathrm{O}$ nível de escolaridade dos pais tem se mostrado como fator decisivo para estabelecer as condições de vida da população ${ }^{23}$. A escolaridade dos pais está associada ao desempenho da criança na alfabetização. Pais com maior escolaridade oferecem maior proteção para a criança por um conhecimento mais estruturado e, teoricamente, por uma renda maior. Pais mais instruídos apresentam maior capacidade para oferecer a seus filhos melhores condições de vida ${ }^{24}$. Os resultados do presente estudo mostraram que o grau de escolaridade materna está associado significativamente com o desempenho escolar da criança. Mau rendimento escolar foi mais frequentemente encontrado em filhos de mães com baixo nível de instrução escolar. Fica evidenciado que a motivação ou o estímulo familiar interferem no desempenho escolar, reforçando ainda mais a importância dos fatores sócioeconômicos no aprendizado. A renda per capita familiar também mostrou associação quanto ao desempenho escolar da criança. Baumann et al. ${ }^{11}$ encontraram associação da latência do P300 com a condição sócio-econômica, provavelmente por estar ligado a maior risco de desnutrição. O P300, nesta pesquisa, não sofreu modificações ligadas aos fatores escolaridade dos pais ou renda per capita. Tal aspecto pode estar relacionado ao fato de que as crianças avaliadas não apresentavam história de desnutrição nos primeiros anos de vida.

Em relação aos traçados eletrencefalográficos, sabe-se que o ritmo se modifica conforme a idade, tanto em repouso como na presença de variados métodos de ativação. À medida que ocorre a maturação cerebral, também ocorre maior organização da atividade bioelétrica, partindo de traçados mais lentos, instáveis e com escassa diferenciação topográfica, para os ritmos mais rápidos e mais diferenciados. O EEG na infância é evolutivo, como também o exame neurológico. A incidência de anormalidades eletrencefalográficas entre as crianças com dificuldades escolares e com problemas 
de conduta tem preocupado vários autores ${ }^{25}$. Rotta ${ }^{26}$ observou que crianças com bom desempenho escolar apresentavam, na sua maioria, traçados eletrencefalográficos normais, sendo que as crianças com dificuldades escolares apresentavam leve predomínio de traçados lentos sobre os normais. Nessa pesquisa , as crianças, mesmo com mau desempenho escolar, apresentavam ritmos de base adequados para a faixa etária, e a lentificação do traçado de base, quando presente, estava associada a alterações paroxísticas. Essa observação se deve provavelmente ao fato de que a média de idade da amostra estudada por Rotta ${ }^{26}$ era inferior à da presente pesquisa. Estudos prévios relataram correlação na modificação da latência do P300 na presença de alterações eletrencefalográficas ${ }^{27}$. Na presente pesquisa, o achado de alterações no EEG, do tipo alterações paroxísticas centro-temporais, também foi freqüente nas crianças não epilépticas. Das crianças com essas alterações, $30 \%$ não tinham história de crises convulsivas. Provavelmente, a incidência de alterações no EEG foi elevada nesta pesquisa, também pelo fato de que os exames foram realizados nesses dois estágios, sono e vigília. Foi possível constatar que a latência do P300 não apresentou diferença significativa na presença ou não de alterações eletrencefalográficas nos casos estudados. Esses achados permitem fazer considerações de que a presença de manifestações paroxísticas no EEG não é determinante de comprometimento cognitivo, principalmente na população estudada, cujas alterações mais frequentes foram paroxismos centro-temporais. A epilepsia benigna infantil com pontas rolândicas no EEG é relacionada com epilepsia idiopática, ocorrendo em $16 \%$ de todos os casos de epilepsia segundo estudo de Heijbel et al. ${ }^{28}$. Cavazzuti ${ }^{29}$, estudando crianças epilépticas, verificou que em $25 \%$ dos casos as crises iniciaram antes dos 12 anos de idade. Sabe-se que a epilepsia benigna da infância é a mais freqüente forma de epilepsia na criança, com excelente prognóstico, e a história pregressa geralmente não revela anormalidades importantes ${ }^{28}$. Konishi et al. ${ }^{13}$ observaram aumento significativo da latência nos pacientes epilépticos em relação aos controles, sendo que o prolongamento foi maior nos pacientes portadores de epilepsia parcial sintomática em todas as idades durante a infância e nos adolescentes que apresentavam epilepsia generalizada idiopática, sugerindo que o distúrbio cognitivo modifica conforme a idade e varia com a síndrome epiléptica.

Neste estudo, foi realizada a comparação das latências do P300 entre as crianças que apresentavam epilepsia com as que não apresentavam, não tendo ocorrido diferença significativa entre os dois grupos. Nas crianças epilépticas, na faixa etária estudada, a alteração eletrencefalográfica mais encontrada foi paroxismo centro-temporal. A epilepsia benigna da infância foi, portanto, o tipo mais frequentemente encontrado, o que concorda com a literatura ${ }^{29}$.

Quando foi realizada a estratificação dos grupos pelo desempenho escolar, em bom e mau rendimento, o grupo das crianças que não apresentava epilepsia e com bom rendimento diferiu significativamente do grupo das crianças com mau rendimento com e sem epilepsia. O grupo das crianças epilépticas com bom rendimento escolar não mostrou uma tendência significativamente definida. Isso demonstra que a epilepsia não modifica a latência do P300, principalmente quando se trata de epilepsia benigna da infância, como é o caso da maioria dos pacientes desta pesquisa. As crianças com bom rendimento escolar apresentaram resultados com diferenças não significativas, mas presentes, quanto ao fato de serem epilépticas ou não, o que sugere que a medicação anticonvulsivante, usada pelas crianças epilépticas, possa ter sido responsável por este resultado. A epilepsia benigna da infância, com foco rolândico, predominou nas crianças avaliadas, o que era esperado para a idade estudada. Como não houve número significativo para os demais tipos de epilepsia, não foi possível relacionar se mudanças no P300 ou no aprendizado poderiam ser influenciadas pela presença de epilepsia ou síndromes epilépticas de difícil controle. Não foi possível selecionar para este estudo inicial crianças epilépticas com déficit cognitivo severo, pois elas não apresentariam condições de colaborar na realização do exame de forma semelhante aos casos controles. A presença de epilepsia por si só não deve ser encarada, portanto, como sinônimo de comprometimento cognitivo.

Muitos investigadores examinaram os efeitos cognitivos das DAE em crianças, mas os resultados diferem e as conclusões são controversas ${ }^{31-33}$. Nesta pesquisa, a DAE mais freqüentemente utilizada no tratamento das crianças epilépticas foi a CBZ com média do P300 de 375,5 ms (DP 63,4 
$\mathrm{ms}$ ), dados semelhantes aos encontrados por Naganuma et al..$^{30}$ nos casos de epilepsia. Não foi possível analisar os efeitos das DAE, pois as demais drogas não foram utilizadas por número significativo de pacientes. Provavelmente um estudo desenhado especificamente para avaliar os efeitos das DAE pudesse mostrar diferentes resultados. Cada DAE não pode ser correlacionada com a latência do P300 ou com o desempenho escolar pelo mesmo motivo. Essa resposta poderá ser discutida em futuras pesquisas com delineamento de estudo de caso controle do próprio paciente. $\mathrm{O}$ P300 poderá assumir um papel fundamental na detecção, localização e compreensão das afecções mentais, este método fisiológico de avaliação cognitiva será capaz de gerar novas e promissoras pesquisas em nosso meio.

\section{Conclusões}

Não foi encontrada repercussão da epilepsia em relação ao potencial evocado cognitivo, P300, na população estudada. A média da latência do P300 obtida nas crianças sem epilepsia foi de 359 ms, com desvio padrão de $57 \mathrm{~ms}$, e $378 \mathrm{~ms}$, com desvio padrão de $61 \mathrm{~ms}$, para as crianças epilépticas. Foi possível definir a latência do P300 em crianças de 10 anos a 11 anos e 11 meses, com média de latência de 336 ms, nas crianças não epilépticas com bom desempenho escolar, não havendo diferença quanto ao gênero.

As crianças não epilépticas com bom rendimento escolar diferiram significativamente, quanto à latência do P300, das com mau desempenho escolar com epilepsia ou não. Foi possível estudar, pelo P300, o comprometimento cognitivo das crianças com baixo desempenho escolar, com e sem epilepsia, tendo sido encontrado aumento significativo da latência do P300.

A presença de alterações paroxísticas no EEG não interferiu no resultado da latência do P300 no grupo estudado. CBZ, como terapia antiepiléptica, não foi capaz de alterar significativamente o P300 na população estudada. A baixa freqüência com que as outras drogas antiepilépticas foram utilizadas, devido à faixa etária estudada, não permitiu determinar sua repercussão na latência do P300.

A escolaridade da mãe e a renda per capita estavam significativamente relacionadas ao desempenho escolar da criança, não interferindo no resultado da latência do P300.

\section{REFERÊNCIAS}

1. Halliday D, Resnick R, Merril J. Fundamentos de física 3. 3Ed. Rio de Janeiro: Livros Técnicos e Científicos Editora, 1994:1-14.

2. Brazier MAB. The emergence of electrophysiology as an aid to neurology. In Aminoff MJ (ed.) Electrodiagnosis in clinical neurology. 3Ed. New York: Churchill Livingstone 1992:1-16.

3. Chiappa KH. In Chiappa KH (ed). Evoked potentials in clinical medicine. 3Ed. Philadelphia: Lippincott-Raven 1997:1-30.

4. Oken BS. Endogenous event-related potentials. In Chiappa KH (ed.) Evoked potentials in clinical medicine. 3Ed. Philadelphia: Lippincott-Raven, 1997:529-564.

5. Pfefferbaum A, Ford JM, Kraemer HC. Clinical utility of long latency "cognitive" event-related potentials (P3): Electroencephalogr Clin Neurophysiol 1990;76:6-12.

6. Goodin DS. Event-related (endogenous) potentials. In Aminoff MJ, (ed.) Electrodiagnosis in clinical neurology. 3Ed. New York: Churchill Livingstone, 1992: 627-48.

7. Meador KJ, Loring DW. Current perspectives on the relationship of the P3 evoked potential and disorders of memory. NNBN 1989;2:161-174.

8. Squires K, Hecox KE. Aplicaciones clínicas de los potenciales evocados endógenos. In Bogacz J (ed.) Los Potenciales evocados en el hombre. Buenos Aires: Ateneo, 1985:120-136.

9. Halgren E, Squires NK, Wilson CL, Rohrbaugh JW, Babb TL, Crandall PH. Endogenous potentials generated in the human hippocampal formation and amygdala by infrequent events. Science 1980;210:803-805.

10. Courchesne E. Neurophysiological correlates of cognitive development: changes in long-latency event-related potencials from childhood to adulthood. Electroencephalogr Clin Neurophysiol 1978;45:468-482.

11. Baumann S, Otto D, Robinson G, Schroeder S, Barton C. The relationship of late positive ERPs, age, intelligence and lead absorption in socioeconomically disadvantaged children. Curr Trends Event-Relat Potent Res 1987; (Suppl 40):617-623.

12. Naganuma Y, Konishi T, Hongou K, Murakami M, Yamatani M, Okada T. Factors affecting P300 latencies in epileptic children. No-To-Hattatsu 1993;25:227-232.

13. Konishi T, Naganuma Y, Hongou K, Murakami M, Yamatani M, Yagi Y. Changes of P300 latency with age in childhood epilepsy. Pediatr Neurol 1995;12:132-135.

14. Martin F, Delpont E, Suisse G, Richelme C, Dolisi C. Long latency event-related potentials (P300) in gifted children. Brain Development 1993;15:173-177. 
15. Aldenkamp AP, Alpherts WCJ, Dekker MJA, Overweg J. Neuropsychological aspects of learning disabilities in epilepsy. Epilepsia 1990;31(Suppl. 4):S9-20.

16. Seidenberg M, Beck N, Geisser M, et al. Academic achievement of children with epilepsy. Epilepsia 1986;27:753-759.

17. Drake M.E, Huber S.J, Pakalnis A, Phillips B.B. Neuropsychological and event-related potential correlates of nonepileptic seizures. Neuroscienses 1993;5:102-104.

18. Guardiola A, Fernandez L.L, Rotta N.T. Um modelo de avaliação das funções corticais. Arq. Neuropsiquiatr. 1989;47:159-164.

19. Mäder M.J. Avaliação neuropsicológica. In Guerreiro CAM, Guerreiro MM. Epilepsia. $2^{\mathrm{a}}$ ed. São Paulo, Lemos Editorial 1996:169-174.

20. Rosemberg S. Encefalopatias crônicas não evolutivas. In Rosemberg S (ed). Neuropediatria. São Paulo: Sarvier, 1992:115-133.

21. Corbett JA. Epilepsy and mental retardation. In Dam M, Gram L, (eds) Comprehensive Epileptology. New York, Raven Press Ltd. 1991:271-280.

22. Ellenberg JH, Hirtz DG, Nelson KB. Do seizures in children cause intellectual deterioration? N Engl J Med 1986;314:1085-1088.

23. IBGE, Instituto Brasileiro de Geografia e Estatística. Pesquisa Nacional por Amostra de Domicílios, 1995.

24. Guardiola A. Estudo das funções corticais em uma amostra representativa de escolares de $1^{\text {a }}$ série de Porto Alegre. Tese Docência Livre de Neurologia, Fundação Faculdade Federal de Ciências Médicas de Porto Alegre. Porto Alegre 1995

25. Rotta NT, Guardiola A. Distúrbios de aprendizagem. In Diament A, Cypel S, (eds.) Neurologia infantil. 3Ed. São Paulo: Atheneu 1996:1062-1074.

26. Rotta NT. Avaliação neurológica evolutiva eletrencefalográfica e psicológica em crianças com rendimento escolar deficiente. Tese - Docência Livre em Neurologia, Fundação Faculdade Católica de Medicina de Porto Alegre: Porto Alegre 1975

27. Meador KJ, Loring DW, Huh K, King DW, Gallager BB. Long-latency evoked potentials during aura of temporal lobe origin. Int J Neurosci 1990;50:127-130.

28. Heijbel J, Blom S, Bergfors PG. Benign epilepsy of children with centrotemporal EEG foci: a study of incidence rate in outpatient care. Epilepsia 1975;16:657-664.

29. Cavazzuti GB. Epidemiology of different types of epilepsy in school age children of Modena, Italy. Epilepsia 1980;21:57-62.

30. Naganuma Y, Konishi T, Hongou K, et al. Auditory event-related potentials in benign childhood epilepsy with centrotemporal spike: the effects of carbamazepine. Clin Electroencephalogr 1994;25:8-12.

31. Chen YJ, Kang WM, So WCM. Comparison of antiepileptic drugs on cognitive function in newly diagnosed epileptic children: a psychometric and neurophysiological study. Epilepsia 1996;37:81-86.

32. Panagopoulos GR, Thomaides T, Tagaris R, Karageorgiou CL. Auditory event related potentials in patients with epilepsy on sodium valproate monotherapy. Acta Neurol Scand 1997;96:62-64.

33. Trimble MR. Antiepileptic drugs, cognitive function, and behavior in children: evidence from recent studies. Epilepsia 1990;31(Suppl. 4):S30-34. 